\title{
Marginal decline in forced vital capacity is associated with a poor outcome in idiopathic pulmonary fibrosis
}

\author{
C.J. Zappala*,\#, P.I. Latsi*,†, A.G. Nicholson*, T.V. Colby`, D. Cramer*, E.A. Renzoni*, \\ D.M. Hansell*, R.M. du Bois* and A.U. Wells*
}

ABSTRACT: In therapeutic studies in idiopathic pulmonary fibrosis (IPF), the low prevalence of significant change in pulmonary functional tests (PFTs) has been a major constraint. The prognostic value of "marginal" changes in PFTs in IPF and fibrotic non-specific interstitial pneumonia (NSIP) was evaluated.

In patients with biopsy-proven IPF $(n=84)$ and NSIP $(n=72)$, forced vital capacity $(F V C)$ and diffusing capacity of the lung for carbon monoxide $(D \mathrm{~L}, \mathrm{CO})$ trends at 6 months were categorised as "significant" (FVC >10\%; DL,CO >15\%) or "marginal" (FVC 5-10\%; DL,CO 7.5-15\%). Proportional hazards analysis and time-dependent receiver operating characteristic methodology were used to examine PFT trends against mortality.

In IPF, reductions in FVC were significant in 22 cases (26\%) and marginal in 19 cases $(23 \%)$. Mortality was higher in patients with a significant decline in FVC (hazard ratio (HR) $2.80,95 \% \mathrm{Cl}$ 1.54-5.06; $p<0.001)$ and those with a marginal decline in FVC (HR 2.31, 95\% Cl 1.19-4.50; $p=0.01)$ than in those with stable disease. Progression-free survival was lower when the decline in FVC was marginal than in stable disease (HR 2.34, 95\% Cl 1.19-4.60; $p=0.01)$. Marginal changes in $D L, C O$ in IPF and marginal changes in FVC and $D L, C O$ in fibrotic NSIP did not provide useful prognostic information.

Marginal change in FVC in IPF denotes a poor outcome. These findings are applicable to clinical practice and to the selection of patients with more progressive disease for therapeutic studies.

KEYWORDS: Forced vital capacity, idiopathic pulmonary fibrosis, marginal decline

n idiopathic pulmonary fibrosis (IPF) and fibrotic non-specific interstitial pneumonia (NSIP), serial changes in pulmonary function tests (PFTs) at 6 or 12 months have had greater prognostic value than baseline data [1-6]. The current threshold values used to define "significant decline" are a reduction from baseline values of $10 \%$ for forced vital capacity (FVC) and $15 \%$ for diffusing capacity of the lung for carbon monoxide (DL,CO) [1]. Decline of a lesser magnitude (marginal) may indicate real disease progression, especially when accompanied by increased symptoms or other evidence. If real disease progression can be defined in terms of marginal PFT thresholds, then their inclusion in clinical decision-making and in therapeutic trial end-points would allow increased recognition of clinically relevant disease behaviour.

The paucity of signal offered by current significant PFT thresholds has been a limitation in trials of new agents. The IFIGENIA (Idiopathic Pulmonary
Fibrosis International Group Exploring $N$-acetylcysteine I Annual) group, investigating $\mathrm{N}$-acetylcysteine in IPF, found a difference of $8 \%$ predicted in FVC and $14 \%$ pred in DL,CO at 12 months between the treatment and placebo groups [7]. Similarly, AzUMA et al. [8], investigating pirfenidone in IPF, found only a preservation of FVC by $0.1 \mathrm{~L}$ between treatment and placebo groups, although this was statistically significant. Controversially, both groups interpreted these marginal results as positive and relevant to clinical practice.

Our hypothesis was that a marginal change in lung function parameters would predict survival and allow a more sensitive signal to be used in clinical decision-making and therapeutic trials. We therefore determined the prognostic significance of relative marginal change in FVC and $D \mathrm{~L}, \mathrm{CO}$ at 6 months in patients with IPF and fibrotic NSIP.

Some of the results of this study have previously been presented in abstract form [9].

\section{AFFLLIATIONS}

*Royal Brompton Hospital and National Heart and Lung Institute, London, UK,

\#Dept of Medicine, University of Melbourne, Victoria, Australia, and "Mayo Clinic, Scottsdale, AZ, USA

\section{CORRESPONDENCE}

A.U. Wells

Interstitial Lung Disease Unit

Royal Brompton Hospital and NHLI Imperial College Emmanuel Kaye Building

1B Manresa Road London SW3 6LP UK

E-mail: Athol.Wells@rbht.nhs.uk.

Received:

Oct 142008

Accepted after revision:

Sept 272009

First published online:

Oct 192009 


\section{MATERIAL AND METHODS}

Between January 1, 1978, and June 30, 2005, 191 patients met the histological criteria at surgical biopsy for IPF $(n=103)$ or fibrotic NSIP $(n=88)$. IPF patients met American Thoracic Society/ European Respiratory Society (ATS/ERS) diagnostic criteria [10]. Clinical criteria for NSIP comprised: 1) bilateral predominantly basal or widespread crackles; 2 ) radiographic evidence of predominantly basal bilateral lung infiltrates with ground-glass or reticular opacities on either chest radiograph or highresolution computed tomography (HRCT) scan from 1991 onwards; 3) a restrictive functional defect or isolated reduction in diffusing capacity; 4) absence of features suggestive of hypersensitivity pneumonitis or organising pneumonia; 5) no other known cause of pulmonary fibrosis or associated disease.

Patients without serial PFT data were excluded (IPF $n=19$; fibrotic NSIP $n=16$ ). Excluded IPF patients were characterised by greater baseline impairment of FVC and DL,CO levels and higher mortality (data not shown). The remaining 156 patients (IPF $\mathrm{n}=84$; fibrotic NSIP $\mathrm{n}=72$ ) comprised the study cohort. Part of the cohort has been reported in outcome studies $[2,11,12]$.

Histological diagnoses of usual interstitial pneumonia or fibrotic NSIP were made by two histopathologists blinded to clinical data $(\kappa=0.53)$, with diagnostic divergences resolved by consensus. In six cases (IPF $n=2$; excluded $n=4$ ), a final diagnosis was made in formal multidisciplinary review (by a histopathologist, radiologist and clinician) [13, 14]. Vital status at October 31, 2006, was determined. Transplanted patients $(n=4)$ were censored as alive at the date of transplant. Treatment regimens included: 1) combination immunosuppressive treatment including low-dose prednisolone $(10 \mathrm{mg})$; or 2) high-dose prednisolone initially (40-60 mg), reducing to a maintenance average dose of $10 \mathrm{mg}$.

Baseline disease severity was quantified using DL,CO and composite physiological index (CPI) levels, based on their superior prognostic value in previous analyses [2, 4, 5, 12, 15] and in preliminary analyses in the current cohort (data not shown).

Serial PFT trends at $6( \pm 2)$ months, expressed as percentages of baseline values, were evaluated for FVC (PKM Spirometer; P.K. Morgan, Kent, UK; or Jaeger Compact; Viasys Healthcare, Warwickshire, UK) and DL,CO (single breath or re-breathing technique, P.K. Morgan respirometer) [16]. Relative trends were defined a priori as significant (FVC $>10 \%$ pred; $D \mathrm{~L}, \mathrm{CO}$ $>15 \%$ pred) [2] or marginal (FVC 5-10\% pred; DL,CO 7.5-15\% pred) compared with baseline. Criteria for marginal decline were chosen to allow rapid computation in clinical practice, reflecting the rationale of current ATS criteria for significant PFT change.

\section{Data analysis}

Group comparisons were made using unpaired t-tests or Chisquared statistics. PFT trends were evaluated against mortality using proportional hazards analysis [17], with findings reexamined in multivariate models, including adjustment for age, sex, smoking status and baseline disease severity (CPI and $D \mathrm{~L}, \mathrm{CO}$ levels in separate models) as follows.

1) Outcome was evaluated from the date of the 6-month follow-up PFTs. Mortality was compared between patient subgroups (significant decline; marginal decline; stable disease) in IPF and NSIP. Progression-free survival (i.e. duration of follow-up to a significant decline in FVC or death) was compared between patient subgroups in IPF.

2) Alternative threshold values for PFT decline were examined against mortality in IPF, comparing patients with marginal decline and those with stable disease. In post hoc analysis, the FVC threshold of $5 \%$ in IPF, corresponding to marginal decline, was compared with alternative thresholds $(3,4,6$ and $7 \%$ ) using proportional hazards analysis. This analysis was performed to identify the optimal FVC trend threshold for possible use in pharmaceutical studies with regard to recruitment of patients with more progressive disease and end-point definition.

3) Survival analyses were repeated in the entire cohort of both IPF and NSIP patients $(n=156)$, with baseline $D \mathrm{~L}, \mathrm{CO}<40 \%$ pred $(n=71)$ and, separately, with $D \mathrm{~L}, \mathrm{CO}>40 \%$ pred $(n=85)$. This subanalysis was performed to explore the hypothesis that the prognostic value of PFT trends is influenced by the severity of baseline functional impairment.

\section{RESULTS}

\section{Baseline demographics and determinants of outcome}

Patients with IPF were older, more often male and more often current or ex-smokers, as shown in table 1. Mortality was higher in IPF (5-yr survival 19\%) than in NSIP (5-yr survival $65 \%)$.

\section{Prevalence of serial decline in DL,CO and FVC}

As shown in table 2, declines in DL,CO and FVC at 6 months were more prevalent in IPF than in NSIP. In IPF, a high proportion of patients had either a significant or marginal decline (DL,CO 55\%; FVC 49\%). In NSIP, fewer patients showed a marginal decline, such that the overall proportion with a significant or marginal decline was only $24 \%$ for $D \mathrm{~L}, \mathrm{CO}$ and $28 \%$ for FVC.

In IPF patients a marginal decline in FVC was confirmed when PFTs were next measured in 16 of 18 cases (88.9\%). One patient died without repeat PFTs.

\section{Mortality in relation to PFT trends}

Mortality was compared between patient subgroups with a significant decline, marginal decline and stable disease. Median survival was 29 months in patients with stable or improved serial FVC trends at 6 months $(n=38), 14$ months in patients with a marginal decline in FVC at 6 months $(n=23)$, and 7 months in patients with a significant decline in FVC at 6 months $(n=23)$. In patients with IPF, mortality was higher with a marginal FVC decline (hazard ratio (HR) $2.31,95 \% \mathrm{CI}$ $1.19-4.50 ; \mathrm{p}=0.01$ ) and with a significant FVC decline (HR 2.80, $95 \%$ CI 1.54-5.06; p<0.001) than in stable disease (fig. 1). As shown in table 3 , this finding was robust with adjustment for disease severity (marginal decline versus stable disease; $\mathrm{p}<0.005)$. Mortality did not differ between patients with a marginal FVC decline and patients with a significant FVC decline.

By contrast, a marginal decline in DL,CO was not associated with increased mortality in IPF (fig. 2). Similarly, a marginal PFT decline (both in FVC and in DL,CO) was not associated 


\begin{tabular}{lcc}
\hline TABLE 1 & $\begin{array}{l}\text { Demographics, baseline clinical data and } \\
\text { selected pulmonary function indices in idiopathic } \\
\text { pulmonary fibrosis (IPF) and non-specific } \\
\text { interstitial pneumonia (NSIP) patients }\end{array}$ \\
\multicolumn{4}{c}{ IPF } \\
\hline NSIP
\end{tabular}

Data are presented as mean \pm SD or $n(\%)$, unless otherwise stated. IPF patients were slightly older with decreased survival and were more likely to be ever smokers and male, otherwise the two disease cohorts had comparable baseline lung function and treatment rates. $D \mathrm{~L}, \mathrm{CO}$ : diffusing capacity of the lung for carbon monoxide; \% pred: \% predicted; FVC: forced vital capacity; CPI: composite physiological index. *: $p<0.05$.

with increased mortality in NSIP. A significant decline in DL,CO and FVC in both disease cohorts was predictive of increased mortality.

In the combined cohort $(n=156)$, a marginal decline in FVC was associated with a poor outcome, both in 71 patients with severe disease (baseline DL,CO <40\%: HR 2.27, 95\% CI 1.28 4.02; $\mathrm{p}<0.005)$ and in 85 patients with less severe disease (baseline DL,CO >40\%: HR 2.98, 95\% CI 1.08-3.60; $\mathrm{p}=0.03$ ).

\section{Progression-free survival in IPF}

As shown in figure 3, progression-free survival was highest in patients with stable disease, and higher in patients with a 5 $10 \%$ decline in FVC than in those with a significant FVC decline (HR 2.24, 95\% CI 1.63-3.08; $\mathrm{p}<0.001$ ). When compared with stable disease, patients with a $5-10 \%$ decline in FVC had a marginal reduction in progression-free survival (HR 1.82, 95\% CI 0.97-3.40; $p=0.06)$, which became statistically significant with adjustment for baseline DL,CO (HR 2.56, 95\% CI 1.17-4.38; $\mathrm{p}=0.02$ ) and CPI levels (HR 2.34, 95\% CI 1.19-4.60; $\mathrm{p}=0.01$ ).

Parallel analyses of DL,CO trends in IPF revealed that progressionfree survival did not differ between patients with stable disease and those with a marginal decline in DL,CO $(p=0.88)$.

\section{Threshold values}

Alternative threshold values for marginal change of between $3 \%$ and $7 \%$ were evaluated for FVC decline in IPF, with the

\begin{tabular}{|c|c|c|c|c|}
\hline \multirow[t]{2}{*}{ TABLE 2} & \multicolumn{4}{|c|}{$\begin{array}{l}\text { Prevalence of serial decline at } 6 \text { months in } \\
\text { idiopathic pulmonary fibrosis (IPF) and non- } \\
\text { specific interstitial pneumonia (NSIP) patients }\end{array}$} \\
\hline & & IPF & NSIP & p-value \\
\hline \multirow{2}{*}{\multicolumn{2}{|c|}{$\begin{array}{l}\text { Subjects n } \\
\text { DL,Co }\end{array}$}} & 84 & 72 & \\
\hline & & & & \\
\hline \multicolumn{2}{|c|}{ Significant decline $>15 \%$} & $23(27)$ & $13(18)$ & 0.13 \\
\hline \multicolumn{2}{|c|}{ Marginal decline $7.5-15 \%$} & $23(27)$ & $4(6)$ & $<0.001$ \\
\hline \multicolumn{2}{|c|}{ Marginal or significant decline $>7.5 \%$} & $46(55)$ & 17 (24) & $<0.001$ \\
\hline \multicolumn{5}{|l|}{ FVC } \\
\hline \multicolumn{2}{|c|}{ Significant decline $>10 \%$} & $22(26)$ & $13(18)$ & 0.19 \\
\hline \multicolumn{2}{|c|}{ Marginal decline 5-10\% } & $19(23)$ & $7(10)$ & 0.06 \\
\hline \multicolumn{2}{|c|}{ Marginal or significant decline $>5 \%$} & $41(49)$ & $20(28)$ & $<0.01$ \\
\hline \multicolumn{5}{|c|}{$\begin{array}{l}\text { Data are presented as } n(\%) \text {, unless otherwise stated. A higher proportion of } \\
\text { patients with IPF had either significant or marginal decline compared with those } \\
\text { with NSIP ( } p<0.001) \text {. D L,CO: diffusing capacity of the lung for carbon monoxide; } \\
\text { FVC: forced vital capacity. }\end{array}$} \\
\hline
\end{tabular}

exclusion of patients with a significant decline in FVC. The prognostic significance of thresholds of 4,5 or $6 \%$ were broadly similar (table 4 ). By contrast, a 3 or $7 \%$ threshold was nondiscriminatory.

The sensitivity and specificity of a marginal and a significant decline in identifying mortality within $1 \mathrm{yr}$ and within 2 yrs is shown in table 5. Marginal change offered advantages over significant change in predicting death within 2 yrs, although the rise in sensitivity from 37 to $65 \%$ was partially offset by a smaller fall in specificity from 85 to $72 \%$.

\section{DISCUSSION}

We report that marginal (5-10\%) declines in FVC at 6 months are linked to increased mortality in IPF. This finding was robust after adjustment for baseline disease severity (using CPI and DL,CO levels in separate models) in subgroup analysis, and when progression-free survival was evaluated as an alternative end-point. The amalgamation of marginal and significant declines in FVC provided an outcome end-point that was positive at 6 months in almost $50 \%$ of IPF patients.

The low prevalence of a marginal change in FVC in fibrotic NSIP removed any possibility of demonstrating a linkage between marginal change and outcome in that disease. However, it can be argued along Bayesian principles that such a linkage is, in any case, less likely in patients with fibrotic NSIP. A decline in FVC of 5-10\% may represent either technical variation in measurement or progression of disease. In IPF, the pre-test probability of disease progression is high and, thus, marginal decline is relatively more likely to denote true deterioration in individual cases, a conclusion that is strongly supported by our findings. By contrast, because NSIP is less progressive than IPF $[1,2,18]$, marginal change in NSIP is necessarily more likely to represent measurement variation.

\section{Relevance to clinical practice}

It should be stressed that, in isolation, marginal FVC trends are not sufficiently prognostically accurate to determine 


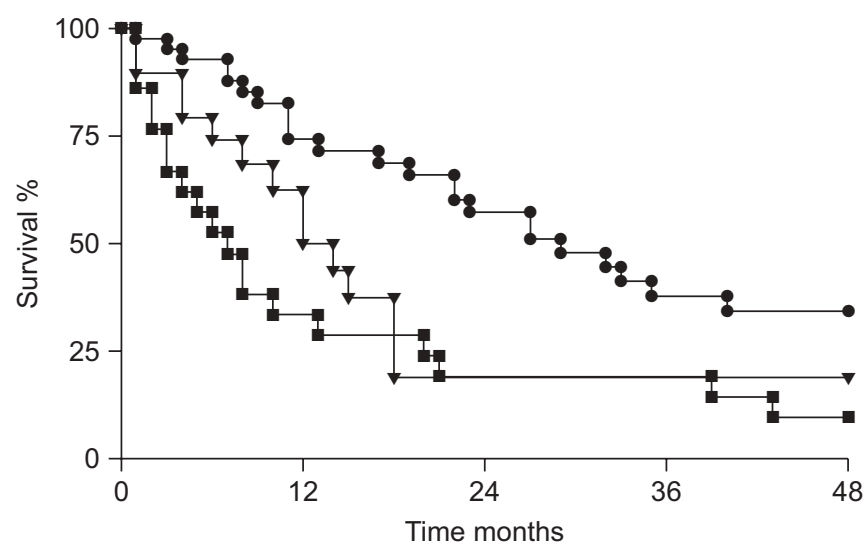

FIGURE 1. 4-yr survival in relation to the magnitude of serial change in forced vital capacity at 6 months in patients with idiopathic pulmonary fibrosis $(n=84)$ Declines of $5-10 \%$ (marginal; $\mathbf{\nabla}$ ) and $>10 \%$ (significant; $\mathbf{~ ) ~ w e r e ~ b o t h ~ a s s o c i a t e d ~}$ with a worse prognosis than stable disease $(\bullet)(p<0.005)$.

management. However, our findings suggest that marginal reductions in serial FVC provide important ancillary support for true disease progression in the context of symptomatic deterioration or equivocal change on chest radiography. In other cases, marginal FVC trends provide a logical indication for more intense evaluation, including the performance of HRCT or repetition of PFTs. In that regard, marginal trends were reproduced in the great majority of patients when PFTs were next performed.

There are also possible implications for routine prognostic evaluation. The use of marginal trend thresholds may identify patients with real disease progression at higher risk of mortality who are not captured by traditional thresholds based on significant decline $[1,2,4,5]$. Clinicians are often confronted with diagnostic ambiguity between fibrotic NSIP and IPF, in the absence of surgical lung biopsy. Serial PFT trends at 612 months provide useful prognostic information, with stability indicative of a better outcome $[2,3,5,12]$. However, our results indicate that patients with IPF should be regarded as stable only if the FVC has declined by $<5 \%$ at 6 months, although it should also be stressed that the long-term outcome remains poor, even in stable IPF, and that a single study is an

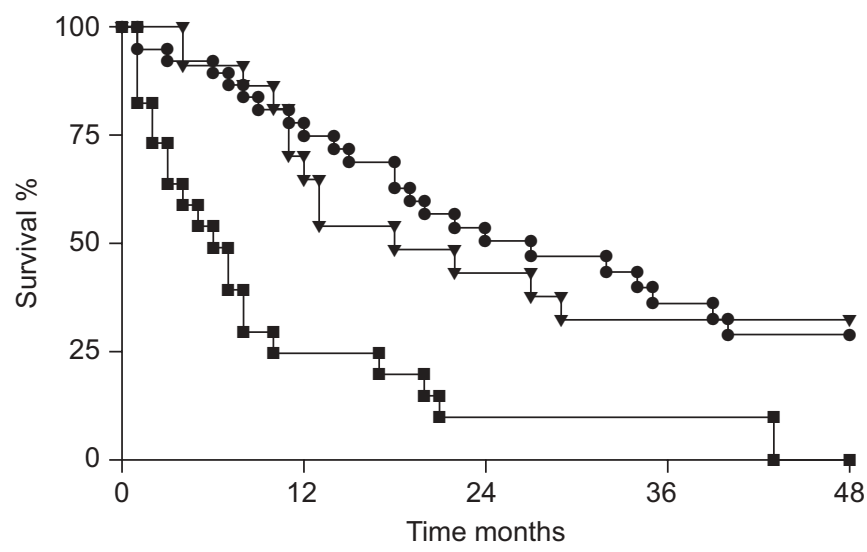

FIGURE 2. 4-yr survival in relation to the magnitude of serial change in diffusing capacity of the lung for carbon monoxide at 6 months in patients with idiopathic pulmonary fibrosis $(n=84)$. Survival did not differ between those with stable disease $(\bullet)$ or a $7.5-15 \%$ (marginal; $\mathbf{\nabla}$ ) decline. Patients with a $>15 \%$ (significant; ) decline had a worse prognosis than those with stable disease or a marginal decline $(p<0.0005)$

insufficient basis on which to change routine clinical recommendations [19].

\section{Relevance to clinical trials}

The criteria used to define marginal decline in the present study were based on the principle that selected cut-off values should facilitate rapid computation in clinical practice. The current ATS criteria for significant change do not exactly capture the reproducibility of individual indices but are a close and user-friendly approximation. However, in pharmaceutical studies in which average population effects are defined rather than change in individual patients, clinical convenience is a lesser consideration and this prompted us to explore lower threshold values for marginal decline in the hope of amplifying the outcome signal. We demonstrated that threshold values of 4,5 and $6 \%$ provided equivalent predictive power, whereas threshold values of 3 and $7 \%$ were non-discriminatory.

These findings have implications for the selection of end-points. In therapeutic trials of pirfenidone [8] and $\mathrm{N}$-acetylcysteine [7] in IPF, average treatment effects were not definitive. With

\section{TABLE 3 Prognostic value of a marginal decline (5-10\% change) in forced vital capacity (FVC) when patients with a significant} decline ( $>10 \%$ change) were excluded, controlled for disease severity

\begin{tabular}{|c|c|c|c|c|}
\hline & \multicolumn{2}{|c|}{$\mathrm{IPF}^{\#}$} & \multicolumn{2}{|c|}{ NSIP" } \\
\hline & HR $(95 \% \mathrm{Cl})$ & p-value & HR $(95 \% \mathrm{Cl})$ & p-value \\
\hline 5-10\% decline FVC univariate & $2.31(1.19-4.50)$ & 0.014 & $1.36(0.40-4.66)$ & 0.62 \\
\hline $5-10 \%$ decline FVC $D \mathrm{~L}, \mathrm{CO}$ controlled & $3.33(1.61-6.88)$ & $<0.001$ & $1.46(0.42-5.00)$ & 0.55 \\
\hline 5-10\% decline FVC CPI controlled & $3.60(1.70-7.62)$ & $<0.001$ & $1.29(0.38-4.42)$ & 0.69 \\
\hline
\end{tabular}

Significant $(p<0.05)$ relationships are shown, with hazard ratios $(H R)$, expressing the difference in risk of mortality between those with change and those without (with $95 \%$ $\mathrm{Cl}$ ). In idiopathic pulmonary fibrosis (IPF), mortality was higher with both marginal and significant decline than in stable disease when controlled for baseline disease severity $(p<0.005)$, with no difference in mortality between patients with a marginal decline in FVC and those with a significant decline in FVC (both $p<0.001)$. A marginal decline in FVC did not predict mortality in non-specific interstitial pneumonia (NSIP). DL,CO: diffusing capacity of the lung for carbon monoxide; CPI: composite physiological index. ${ }^{*}: n=84 ; ": n=72$. 


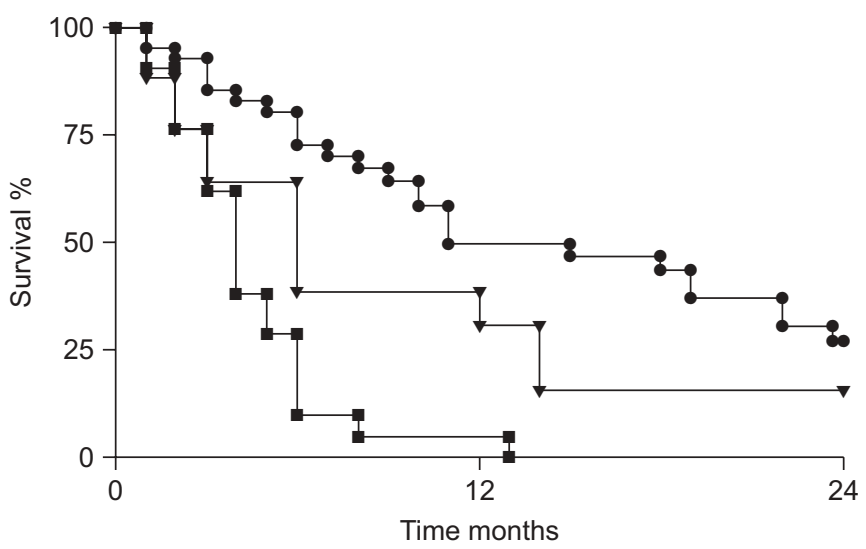

FIGURE 3. 2-yr progression-free survival in relation to the magnitude of forced vital capacity (FVC) serial change at 6 months in idiopathic pulmonary fibrosis $(n=84)$. A decline of $5-10 \%$ (marginal; $\mathbf{\nabla})$ or $>10 \%$ (significant; $\mathbf{\square})$ is associated with worse prognosis than stable disease $(\bullet)(p<0.005)$. Patients with marginal decline had an intermediate progression-free survival compared with those with stable disease or a significant decline in FVC

pirfenidone treatment, the relative FVC benefit was only $0.13 \mathrm{~L}$ at 9 months [8]. $\mathrm{N}$-acetylcysteine therapy was associated with a relative improvement of $8 \%$ in FVC and $14 \%$ in DL,CO [7]. These average effects are deceptive because they are not uniform but represent a clear benefit in some but not all cases. Our findings underline the possibility that even marginal FVC benefits in individuals may represent a worthwhile therapeutic effect (although the degree to which the 6-month trends in our study can be equated to 12 -month trends in clinical trials is unclear). However, surrogate end-points are not fully validated by a single study [19], and independent verification of disease progression may be required. Serial HRCT, which has yet to be validated as an independent outcome variable in IPF, may have a valuable role in providing collateral morphological evidence of disease progression. A combined end-point of marginal FVC decline and progression of disease on HRCT (or, alternatively, declining exercise tolerance) merits further study.

More importantly, our findings have implications for the selection of patients for enrolment in clinical trials. Current end-points in therapeutic studies may not be sufficiently sensitive. In a retrospective analysis of a placebo-controlled trial of interferon- $\gamma$, the primary end-point (change in FVC, change in alveolar-arterial oxygen tension gradient or death) was met in $<30 \%$ of cases [20]. By implication, a prohibitively large number of patients would be required to demonstrate a worthwhile partial treatment effect. It can be argued that patients with more indolent disease tend to be selectively enrolled in placebo-controlled studies in diffuse lung disease [21-23]. Our results indicate that an enrolment criterion of marginal or significant decline in FVC during the 6 months before entry into a clinical trial, selects patients more likely to meet a progression-free survival end-point during a treatment period of 1-2 yrs. However, as in the detection of disease progression in clinical practice, it appears likely that the optimal algorithm for patient selection, when refined in future studies, will consist of a combination of marginal FVC trends and other ancillary evidence of disease progression, including

\begin{tabular}{|llc}
\hline TABLE 4 & $\begin{array}{l}\text { Proportional hazards comparative analysis of } \\
\text { marginal forced vital capacity (FVC) thresholds in } \\
\text { idiopathic pulmonary fibrosis, with the exclusion } \\
\text { of patients with a significant decline in FVC }\end{array}$ \\
\hline FVC decline & HR (95\% Cl) & p-value \\
\hline 7\% threshold & $1.76(0.86-3.60)$ & 0.12 \\
$\mathbf{6 \%}$ threshold & $2.08(1.05-4.13)$ & 0.04 \\
$\mathbf{5 \%}$ threshold & $2.31(1.19-4.50)$ & 0.01 \\
$\mathbf{4 \%}$ threshold & $2.11(1.11-4.00)$ & 0.02 \\
$\mathbf{3} \%$ threshold & $1.43(0.75-2.68)$ & 0.27 \\
\hline
\end{tabular}

Significant $(p<0.05)$ relationships are shown, with hazard ratios $(H R)$, expressing the change in risk of mortality between those with change and those without change (with $95 \% \mathrm{Cl}$ ). The prognostic significance of thresholds of 4 and $5 \%$ did not differ. By contrast, a $3 \%$ threshold was always inferior to 4 and $5 \%$ thresholds.

symptomatic decline and/or evidence of disease progression on HRCT.

\section{Limitations of the study}

Selection bias is an important consideration because of possible over-representation of NSIP in this and other studies, due to the exclusion of IPF patients in whom typical HRCT appearances obviated biopsy. Furthermore, although histological evaluation provided diagnostic security and allowed a comparison between IPF and fibrotic NSIP, biopsied patient cohorts are known to be younger and to have less severe

\begin{tabular}{|c|c|c|}
\hline TABLE 5 & \multicolumn{2}{|c|}{$\begin{array}{l}\text { Prognostic value of marginal decline }(5-10 \% \\
\text { change) and significant decline ( }>10 \% \text { change) } \\
\text { in forced vital capacity (FVC) in idiopathic } \\
\text { pulmonary fibrosis ( } n=84) \text {, based on the } \\
\text { sensitivity and specificity of these thresholds for } \\
\text { death within } 1 \text { and } 2 \text { yrs }\end{array}$} \\
\hline FVC decline & 12 months & 24 months \\
\hline \multicolumn{3}{|l|}{$5 \%$ threshold } \\
\hline Sensitivity & 83 (63-95); 15/18 & 65 (51-78); 28/43 \\
\hline Specificity & 61 (49-72); 40/66 & 72 (57-84); 28/39 \\
\hline PPV & 37 (23-52); 15/41 & 72 (57-84); 28/39 \\
\hline NPV & 93 (83-98); 40/43 & 65 (50-78); 28/43 \\
\hline LR & $2.12(1.47-3.04)$ & 2.31 (1.34-3.99) \\
\hline \multicolumn{3}{|c|}{$10 \%$ threshold } \\
\hline Sensitivity & 67 (45-85); 12/18 & 37 (24-52); 16/43 \\
\hline Specificity & 85 (75-92); 56/66 & 85 (71-94); 33/39 \\
\hline PPV & 55 (35-73); 12/22 & 73 (53-88\%); 16/22 \\
\hline NPV & 90 (81-96); 56/62 & 55 (43-67\%); 33/60 \\
\hline LR & $4.4(2.28-8.49)$ & $2.42(1.05-5.56)$ \\
\hline
\end{tabular}

Data are presented as \% $(95 \% \mathrm{Cl})$; proportion of patients. Likelihood ratio (LR) data are are presented as $\%(95 \% \mathrm{Cl})$. PPV: positive predictive value; NPV: negative predictive value. The prognostic advantage of marginal change lay in increased sensitivity, particularly for death within 2 yrs. Predictive values and LRs are shown for comparison. 
functional impairment [24]. However, serial FVC trends are known to have a greater prognostic value in less severe disease (as judged by lack of desaturation on a baseline walk test) [3]. Thus, marginal trends in FVC may be less prognostically useful in IPF patients with advanced disease.

The recent widespread introduction of $N$-acetylcysteine therapy as part of standard treatment for IPF and severe fibrotic NSIP had the potential to confound our results, based upon findings in the IFIGENIA study [7]. This consideration prompted us to limit the date of presentation to June 2005. In a small percentage of patients, $\mathrm{N}$-acetylcysteine was introduced during follow-up in the last year of the follow-up period to October 2006. However, it is unlikely that this had a major effect on our findings as it is known that mortality is not significantly influenced by anti-oxidant therapy during the first year of treatment [7].

It should also be stressed that the results of this study are more directly applicable to clinical practice than to therapeutic trials, which generally last for 12 months in IPF. Although 6-month follow-up was almost always possible in surviving patients in our cohort, follow-up at 12 months was highly variable (especially in the last 5 years of the study, with the advent of a "shared care" algorithm with local chest physicians). Thus, an evaluation of the prognostic significance of a 12-month functional trend (as in an earlier study containing some patients in the current study [2]) was no longer practicable in this extended population. Our findings need to be reproduced in another cohort, with follow-up extended to 12 months, to validate marginal change in FVC as a primary end-point in pharmaceutical studies.

Progression-free survival provides an additional outcome variable but represents a blunt end-point when compared with mortality as it depends upon the timing of routine monitoring and opportune lung function testing. By contrast, vital status is generally collated monthly. This factor is unlikely to have materially influenced our results in IPF patients as follow-up PFTs were performed 3-6-monthly in almost all cases.

In conclusion, short-term marginal changes in FVC predict a poor outcome in patients with IPF. Our results have important implications for clinical decision-making and recruitment into clinical therapeutic trials.

\section{STATEMENT OF INTEREST}

A statement of interest for A.G. Nicholson can be found at www.erj. ersjournals.com $/ \mathrm{misc} /$ statements.dtl

\section{REFERENCES}

1 American Thoracic Society/European Respiratory Society, American Thoracic Society/European Respiratory Society international multidisciplinary consensus classification of the idiopathic interstitial pneumonias. Am J Respir Crit Care Med 2002; 165: 277-304

2 Latsi PI, du Bois RM, Nicholson AG, et al. Fibrotic idiopathic interstitial pneumonia. The prognostic value of longitudinal functional trends. Am J Respir Crit Care Med 2003; 168: 531-537.

3 Flaherty KR, Andrei AC, Murray S, et al. Idiopathic pulmonary fibrosis. Prognostic value of changes in physiology and six-minute walk test. Am J Respir Crit Care Med 2006; 174: 803-809.
4 Collard HR, King TE, Bartelson BB, et al. Changes in clinical and physiologic variables predict survival in idiopathic pulmonary fibrosis. Am J Respir Crit Care Med 2003; 168: 538-542.

5 Jegal Y, Kim DS, Shim TS, et al. Physiology is a stronger predictor of survival than pathology in fibrotic interstitial pneumonia. Am J Respir Crit Care Med 2005; 171: 639-644.

6 Hanson DH, Winterbauer RH, Kirtland SH, et al. Changes in pulmonary function test results after 1 year of therapy as predictors of survival in patients with IPF. Chest 1995; 108: 305-310.

7 IFIGENIA Study Group. High-dose acetylcysteine in idiopathic pulmonary fibrosis. N Engl J Med 2005; 353: 2229-2242.

8 Azuma A, Nukiwa T, Tsuboi E, et al. Double-blind, placebocontrolled trial of pirfenidone in patients with idiopathic pulmonary fibrosis. Am J Respir Crit Care Med 2005; 171: 1040-1047.

9 Zappala CJ, Latsi PI, Nicholson AG, et al. Marginal declines in FVC levels are associated with increased mortality in IPF but not in NSIP. Am J Respir Crit Care Med 2007; 175: A143.

10 American Thoracic Society/European Respiratory Society. ATS statement. Idiopathic pulmonary fibrosis: diagnosis and treatment. International consensus statement. Am J Respir Crit Care Med 2000; 161: 646-664.

11 Nicholson AG, Fulford LG, Colby TV, et al. The relationship between individual histologic features and disease progression in idiopathic pulmonary fibrosis. Am J Respir Crit Care Med 2002; 166: 173-177.

12 Wells AU, Desai SR, Rubens MB, et al. Idiopathic pulmonary fibrosis: a composite physiologic index derived from disease extent observed by computer tomography. Am J Respir Crit Care Med 2003; 167: 962-969.

13 Lok S. Interstitial lung disease clinics for the management of idiopathic pulmonary fibrosis: a potential advantage to patients. Heart Lung Transplant 1999; 18: 884-890.

$14 \mathrm{Du}$ Bois RM. Evolving concepts in the early and accurate diagnosis of idiopathic pulmonary fibrosis. Clin Chest Med 2006; 27: Suppl. 1, S17-S25.

15 Mogulkoc N, Brutsche MH, Bishop PW, et al. Pulmonary function in idiopathic pulmonary fibrosis and referral for lung transplantation. Am J Respir Crit Care Med 2001; 164: 103-108.

16 Quanjer PH, Tammeling GJ, Cotes JE, et al. Report of working party. Standardized lung function testing. Official statement of the European Respiratory Society. European Community of Steel and Coal. Eur Respir J 1993; 16: 1-100.

17 Kleinbaum DG, Kupper LL, Muller R. Applied regression analysis and other multivariate methods. Boston, Kent, 1988.

18 Martinez FJ. Idiopathic interstitial pneumonias. Usual interstitial pneumonia $v s$ nonspecific interstitial pneumonia. Proc Am Thorac Soc 2006; 3: 81-95.

19 Fleming TR, DeMets DL. Surrogate end points in clinical trials: are we being misled? Ann Intern Med 1996; 125: 605-613.

20 King TE, Safrin S, Starko KM, et al. Analyses of efficacy end points in a controlled trial of interferon- $\gamma 1 \mathrm{~b}$ for idiopathic pulmonary fibrosis. Chest 2005; 127: 171-177.

21 Baughman RP, Drent $M$, Kaburu $M$, et al. Infliximab therapy in patients with chronic sarcoidosis and pulmonary involvement. Am J Respir Crit Care Med 2006; 174: 795-802.

22 Tashkin DP, Elashoff R, Clements PJ, et al. Cyclophosphamide versus placebo in scleroderma lung disease. N Engl J Med 2006; 354: 2655-2666.

23 Hoyles RK, Ellis RW, Wellsbury J, et al. A multicenter, prospective randomized, double-blind placebo-controlled trial of corticosteroids and intravenous cyclophosphamide followed by oral azathioprine for the treatment of pulmonary fibrosis in scleroderma. Arthritis Rheum 2006; 54: 3962-3970.

24 Wells AU, Cullinan P, Hansell DM, et al. Fibrosing alveolitis associated with systemic sclerosis has a better prognosis than lone cryptogenic fibrosing alveolitis. Am J Respir Crit Care Med 1994; 149: 1583-1590. 